\title{
On the isothermality of solar plasmas
}

\author{
E. Landi \\ Artep, Inc at Naval Research Laboratory, 4555 Overlook Ave. S.W., 20375-5320, \\ Washington $D C$ \\ and \\ Department of Ocean, Atmospheric and Space Science, University of Michigan, Ann Arbor, \\ Mi 48109 \\ J.A. Klimchuk \\ NASA Goddard Space Flight Center, Greenbelt, Maryland 20771 USA
}

\begin{abstract}
Recent measurements have shown that the quiet unstructured solar corona observed at the solar limb is close to isothermal, at a temperature that does not appear to change over wide areas or with time. Some individual active loop structures have also been found to be nearly isothermal both along their axis and across their cross-section. Even a complex active region observed at the solar limb has been found to be composed of three distinct isothermal plasmas. If confirmed, these results would pose formidable challenges to the current theoretical understanding of the thermal structure and heating of the solar corona. For example, no current theoretical model can explain the excess densities and lifetimes of many observed loops if the loops are in fact isothermal. All of these measurements are based on the so-called emission measure (EM) diagnostic technique that is applied to a set of optically thin lines under the assumption of isothermal plasma. It provides simultaneous measurement of both the temperature and EM. However, no study has ever been carried out to quantify the uncertainties in the technique and to rigorously assess its ability to discriminate between isothermal and multithermal plasmas. Such a study is the topic of the present work. We define a formal measure of the uncertainty in the EM diagnostic technique that can easily be applied to real data. We here apply it to synthetic data based on a variety of assumed plasma thermal distributions, and develop a method to quantitatively assess the degree of multithermality of a plasma.
\end{abstract}

Subject headings: Sun: corona - Plasma: diagnostic techniques - Sun: UV, EUV, $\mathrm{X}$-ray radiation 


\section{Introduction}

In the past 15 years the launch of many space missions devoted to the study of the Sun such as SOHO, TRACE and Hinode has renewed the interest in the thermal structure of the solar corona and of the plasma structures that populate it, by providing observations of unprecedented quality and resolution. The knowledge of the thermal structure of the upper solar atmosphere is key to unveiling the physical process(es) that heat the solar coronal plasmas to one or more million degrees, even at very low heights above the limb.

The narrow-band images and the spectra obtained with SOHO, TRACE and Hinode have allowed several detailed studies of the thermal structure of solar plasmas at all scales, from individual plasma loops to the large-scale corona. Many authors, using the CDS and SUMER spectrometers on board SOHO, have found that the plasma in the unstructured quiet solar corona is nearly isothermal (Feldman et al. 1999, Warren 1999, Landi et al. 2002). Landi et al. (2006) also found that a large quiet Sun area $\left(0.5 \mathrm{R}_{\odot} \times 1.8 \mathrm{R}_{\odot}\right)$ outside the west limb was also close to isothermal. Landi \& Feldman (2008) have even shown that the plasma of an active region observed at the solar limb was made of three almost isothermal components.

More recent measurements with the Hinode/EIS spectrometer have also shown that the thermal structure of the off-disk quiet corona has an additional, non-negligible tail at higher temperatures (Warren \& Brooks 2009, Brooks et al. 2009). These authors even determined that the temperature dependence of the thermal distribution of quiet Sun coronal plasmas is very similar in 45 different datasets taken during a four months period in 2007, even if the absolute value of the emission measure changes significantly. Such a hot tail is extremely important for theories of coronal heating based on impulsive release of energy, since these predict a small but significant amount of hot plasma to be produced (Cargill \& Klimchuk 2004, Klimchuk et al. 2008, Patsourakos \& Klimchuk 2009). The mere presence of such a hot tail, as well as its properties, can provide important constraints on the heating of the quiet, "background" solar corona.

One of the most widely used diagnostic methods for determining the plasma thermal structure is the emission measure (EM) technique, sometimes called the EM loci technique. We discuss the technique in detail below. Briefly, it is an attempt to find a single temperature and emission measure pair that correctly predicts the intensities of several different spectral lines. The technique has a big advantage over line ratios in that it uses all the lines in a given data set simultaneously so that it is easy to identify any line that is blended or has atomic physics problems. The technique assumes that the observed plasma is isothermal, so whenever it fails, one may conclude that multithermal conditions exist. A primary goal of our work is to quantitatively evaluate the uncertainties in the method and thereby better 
assess its diagnostic potential.

A similar attempt has been made by Judge (2010), who discussed the accuracy of temperature diagnostics with spectral lines and concluded that isothermal conditions can be determined to no better than $\Delta \log T=0.13$ due to uncertain atomic physics. That is, a true delta function in temperature cannot be distinguished from a thermal distribution of width $\Delta \log T=0.13$. He assumed an uncertainty of $20 \%$ in the atomic physics parameters, which he suggested is a lower limit.

Differential emission measure (DEM) analysis is another method that is often applied to plasmas that are known to be multithermal (see for example the review of Phillips et al. 2008). It is an inversion method that uses multiple spectral lines to determine how the plasma is distributed in temperature. However, standard DEM diagnostic techniques experience difficulties in reproducing the sharp variations of the amount of material as a function of temperature that is typical of plasmas with narrow temperature distributions.

In this work we assess the robustness of the EM diagnostic technique for determining whether a plasma is isothermal. We also propose an extension of the technique that allows us to determine the thermal width of more general distributions which is particularly useful for plasmas with narrow temperature distributions. We discuss quantitative measures for answering the following questions:

1. Is a given set of spectral line observations consistent with the emitting plasma being isothermal?

2. If so, what is the maximum thermal width that is allowed by the uncertainties?

3. Must the observed plasma be multithermal?

4. If so, what is the range of possible thermal widths that are allowed by the uncertainties?

Our approach is as follows. We first define a quantitative measure of the uncertainty in the technique, which can and should be used by other researchers. We then apply the technique to simulated intensities of spectral lines emitted by ions formed over a wide range of temperatures, calculated using a variety of ad hoc plasma thermal distributions. By comparing the simulated measurements with the uncertainty, we arrive at some general conclusions about the ability of the technique to constrain the plasma distribution, both in isothermal and multithermal conditions.

The diagnostic technique and the simulated spectra that we will use are introduced in Section 2, while the results of our exercise are reported in Section 3 and discussed in Section 4. 


\section{Method}

\subsection{Diagnostic technique}

The EM formalism was first introduced by Pottasch (1963) and since then it has become a standard method of analysis. We have implemented it following Landi et al. (2002). The intensity $I_{i}$ of an optically thin emission line observed at distance $d$ can be written as

$$
I_{i}=\frac{1}{4 \pi d^{2}} \int_{V} G_{i}\left(T, N_{e}\right) N_{e}^{2} d V
$$

where $N_{e}$ is the electron number density, $V$ is the emitting volume along the line of sight, and $G_{i}\left(T, N_{e}\right)$ is the contribution function of the emitting line. The subscript refers to a line in the dataset, not an energy level. If the electron density $\left(N_{e}\right)$ and temperature $\left(T_{c}\right)$ are constant in the emitting volume along the line of sight, we have

$$
I_{i}=\frac{G_{i}\left(T_{c}, N_{e}\right)}{4 \pi d^{2}} E M \quad E M=\int_{V} N_{e}^{2} d V=N_{e}^{2} V
$$

where EM is the Emission Measure of the plasma. In this case the Emission Measure can be directly evaluated as:

$$
E M=4 \pi d^{2} \frac{I_{i}}{G_{i}\left(T_{c}, N_{e}\right)}
$$

Under the assumption of constant $\mathrm{N}_{e}$ and $\mathrm{T}_{c}$ within the emitting region, this quantity is the same for all the observed lines. The diagnostic method consists of calculating the function $E M(T)$ defined as

$$
E M_{i}(T)=4 \pi d^{2} \frac{I_{i}}{G_{i}\left(T, N_{e}\right)} \quad \Longrightarrow \quad E M_{i}\left(T_{c}\right)=E M
$$

as a function of electron temperature, using the observed intensities $I_{i}$ of each line and a value of the electron density derived from line intensity ratios. In the case of an isothermal plasma, when all the $E M(T)$ curves are displayed in the same plot as a function of temperature, they should intersect at a common point at $\left(T_{c}, E M\right)$. An example of this technique is 
given in Figure 1, where it is applied to simulated spectral line intensities obtained from the CHIANTI database (Dere et al. 1997, 2009, see next Section) assuming an isothermal plasma at $10^{6} \mathrm{~K}$.

In reality, of course, the curves never intersect at a single point even if the plasma is perfectly isothermal. Uncertainties due to photon counting statistics, atomic data problems, intensity calibration errors, incorrect element abundances, or unidentified blends cause the curves to shift up and down, so that there is a finite region through which all or most of the curves pass. The crossing point at the center of this region is identified subjectively and determines the plasma temperature $T_{c}$ and $E M$. The interpretation is complicated, however, because the curves will also deviate from a single intersection point if the the plasma is not actually isothermal, even if the measurements are perfect. The challenge is to determine the range of possible thermal distributions, perhaps including isothermal, that are compatible with the observations given the uncertainties.

\subsection{Measure of isothermality}

To begin to answer these questions, we first define a quantitative measure of the degree to which $\operatorname{EM}(T)$ curves cluster together. Patsourakos \& Klimchuk (2007) did something very similar in an analysis of TRACE triple filter observations. Following their approach, we define the quantities

$$
\begin{aligned}
F(T) & =\frac{1}{M} \Sigma_{i, j}\left|\log \frac{E M_{j}(T)}{E M_{i}(T)}\right| \\
F_{\text {min }} & =\min [F(T)]
\end{aligned}
$$

where $E M_{j}(T)$ and $E M_{i}(T)$ are the EM curves obtained using the observed intensities of lines $j$ and $i$, respectively, and the summation is carried out over the $M=N(N-1) / 2$ possible line pairs $i, j$ available in a dataset of $N$ lines without repetitions (i.e., a given pair is used only once, regardless of which line appears in the numerator). If all lines cross exactly in the same point with temperature $T_{c}$, then $F_{\min }=F\left(T_{c}\right)=0$. If the plasma is multithermal, $F(T)$ will never be zero at any $T$.

Uncertainties in the $E M_{j}(T)$ and $E M_{i}(T)$ are propagated into $F(T)$ and cause this quantity to be uncertain by an amount $\Delta F$. In principle, $\Delta F$ is a function of temperature, but for convenience and since most of the uncertainty sources (see below) are by their own nature independent of temperature, we take it to be constant. As a result of the finite 
uncertainties, $F_{\min }$ will be larger than zero even for plasmas that are isothermal. In order to be consistent with isothermal, the observations must fulfill the condition

$$
F_{\min } \leq \Delta F
$$

If $F_{\text {min }}$ exceeds $\Delta F$, then the deviation from zero cannot be due to measurement errors alone, and there must be a finite spread in the thermal distribution. If $F_{\min }$ is less than $\Delta F$, then isothermal conditions are a valid possibility, and the temperature interval given by $F(T) \leq \Delta F$ defines a range of allowed isothermal temperatures.

There is also the possibility that a multithermal plasma provides a value of $F_{\min }$ that is smaller than $\Delta F$. The data are then unable to distinguish between isothermal and multithermal conditions. The thermal width of the broadest distribution for which $F_{\min }=\Delta F$ under the assumption of zero uncertainties can be taken as a measure of the temperature resolution of the EM technique. Larger experimental uncertainties raise the value of $\Delta F$, and thus they decrease the ability of the EM technique to discriminate between genuinely isothermal plasmas and multithermal plasmas with narrow temperature distributions. Such effects can be mitigated by increasing the number of lines $N$ used in the analysis and using lines from ions formed at very different temperature regimes, as shown below.

The value of $\Delta F$ is calculated from the uncertainties in the $E M(T)$ curves. If we indicate such uncertainties as $\Delta E M$, the maximum possible value of $\Delta F$ for an isothermal plasma at temperature $T_{c}$ is given by

$$
\begin{aligned}
\Delta F & \leq \frac{1}{M} \Sigma_{i, j}\left|\log \frac{E M_{j}\left(T_{c}\right)+\Delta E M_{j}}{E M_{i}\left(T_{c}\right)-\Delta E M_{i}}\right| \\
& =\frac{1}{M} \Sigma_{i, j}\left|\log \frac{1+\Delta_{j}}{1-\Delta_{i}}\right|
\end{aligned}
$$

where $\Delta_{j}=\Delta E M_{j} / E M_{j}\left(T_{c}\right)$ is the relative uncertainty of the $E M_{j}(T)$ curve. Thus, once $\Delta_{i}$ are known, the value of $\Delta F$ can be calculated even before determining the values of the $E M(T)$ themselves.

Since $\Delta I$ and $\Delta G(T)$ errors are not correlated, the uncertainty $\Delta E M_{i}$ in each individual measurement is given by

$$
\left(\frac{\Delta E M_{i}}{E M_{i}}\right)^{2}=\left(\frac{\Delta I_{i}}{I_{i}}\right)^{2}+\left(\frac{\Delta G_{i}}{G_{i}}\right)^{2}
$$


The intensity uncertainty $\Delta I$ is due to two factors: photon counting statistics and photometric calibration, both independent of temperature. Since $F(T)$ is defined as ratios of $E M_{i}(T)$ curves, the absolute intensity calibration plays no role. This was a primary motivation for defining $F(T)$ in this way. Inaccurate relative intensity calibration (i.e., instrumental factors that affect two spectral lines differently) will introduce a systematic error that is difficult to assess. Uncertainties in photon counting statistics can be minimized by using bright lines.

The uncertainties $\Delta G$ are due to two different main sources: atomic physics calculations of transition rates and element abundances. If we modify Equation 4 to separate the elemental abundances $A$ from the rest of the atomic physics,

$$
E M_{i}(T)=\frac{I_{i}}{A_{i} G_{i}\left(T, N_{e}\right)}
$$

then

$$
\left(\frac{\Delta E M_{i}}{E M_{i}}\right)^{2}=\left(\frac{\Delta I_{i}}{I_{i}}\right)^{2}+\left(\frac{\Delta G_{i}}{G_{i}}\right)^{2}+\left(\frac{\Delta A_{i}}{A_{i}}\right)^{2}
$$

$\Delta G / G$ is typically assumed to be in the $15-30 \%$ range and it may depend on temperature if uncertainties affect ion fractional abundances or collision excitation rate coefficients. $\triangle A / A$ only affects ratios of $E M(T)$ curves of different elements and is determined largely by the FIP effect, which can vary between a factor 2 and 4 in different plasmas and is independent of temperature. However, the relative abundances are much less variable within each FIP class (low-FIP or high-FIP - Feldman \& Laming 2000). If the error in the assumed FIP effect is sufficiently large (i.e. a factor 1.5 or more, see below), it is easily detectable through the crossing points in the $E M(T)$ versus $T$ plots obtained with lines of ions belonging to different FIP classes. It is then possible to correct the assumed abundances. In order to check this, we recommend that the EM analysis be carried out on two subsets of lines, each including all the lines belonging to the same FIP class. From the two EM values so obtained, any offset between the abundances of the low- and high-FIP elements can be corrected, and the EM technique can be applied again to the full set of lines. The uncertainties in the relative elemental abundances, once the FIP effect is taken care of, are therefore relatively small. With this method it is possible to correct for FIP effect errors larger than a certain lower limit given by the uncertainties in the EM. This lower limit is determined by the other sources of uncertainty $\Delta I$ and $\Delta G$, and typically is a factor $1.5(\simeq 0.2$ in the $\log$ ).

Estimating the atomic physics uncertainties is extremely difficult, because the uncertainties in the individual radiative and collisional transition rates are not known with precision, 
and it is almost impossible to propagate them through the calculation of level populations and line intensities. A detailed analysis of the effect of errors in the atomic physics parameters is difficult to carry out, because we do not know a priori the probability distributions for the errors. One possible way of estimating how an assumed set of uncertainties in the atomic rates propagates into line intensities and $E M(T)$ curves is to carry out extensive Monte Carlo simulations assuming a variety of reasonable errors for each atomic parameter in the calculation. However, such an extensive set of calculations is beyond the scope of the present work and is deferred to a future paper; here we limit ourselves to a generic $30 \%$ uncertainty to the contribution function of each line at any temperature: this uncertainty level is fairly typical in the literature.

Assuming that $\Delta I / I$ and $\Delta A / A$ are both negligible, Equation 7 gives $\Delta F \simeq 0.27$ for a hypothetical dataset of 15 lines, all with the same $30 \%$ level of uncertainty. We caution, however, that anyone using this equation should determine $\Delta F$ based on their own dataset. In the following section, we consider 13 lines with total uncertainties of $10 \%$ and $40 \%$, for which $\Delta F \simeq 0.09$ and 0.37 , respectively.

\subsection{Simulated spectra and Monte Carlo simulations}

We have applied the EM diagnostic to a set of line intensities calculated using the CHIANTI database (Dere et al. 1997, 2009). The lines that we have considered are listed in Table 1 and are formed at a temperature range spanning the transition region $\left(10^{5} \mathrm{~K}\right)$ to the hot corona $\left(3-4 \times 10^{6} \mathrm{~K}\right)$ typical of active regions in the solar disk. We chose strong lines routinely observed by SOHO/CDS, since many of the studies of the thermal structure of the corona were carried out using CDS. The calculations were performed assuming the electron density $\mathrm{N}_{e}=10^{9} \mathrm{~cm}^{-3}$, and adopting the ion abundances from Mazzotta et al. (1998) and the coronal element abundances of Feldman et al. (1992).

We wish to determine the range of thermal widths that are consistent with an $F_{\min }$ constructed from real observations. This depends on both the detailed shape of the thermal distribution and the measurement uncertainties. We consider two idealized possibilities for the distribution shape. First, we use a Gaussian in $\log T$, peaked at $\log T_{0}$ with amplitude $A_{0}$ and width $\sigma$ :

$$
\varphi(\log T)=A_{0} e^{-\frac{\left(\log T-\log T_{0}\right)^{2}}{\sigma^{2}}}
$$

where $\varphi(\log T)$ is the differential emission measure of a plasma. We have defined the DEM such that $\varphi(\log T) d \log T$ is the emission measure contained in logarithmic temperature in- 
terval $d \log T$ and has units of $\mathrm{cm}^{-3}$. We choose $\log T_{0}=6.0$ as the centroid for all of our distributions. This value is very close to the typical temperature of off-disk quiet Sun plasmas. We allow $\sigma$ to vary and adjust $A_{0}$ so that the total integrated $\mathrm{EM}$ is $10^{27} \mathrm{~cm}^{-3}$. The magnitude $A_{0}$ is unimportant, however, as long as it is large enough to justify our neglect of line intensity uncertainties. The full width at half maximum (FWHM) of the distribution, designated $\Delta \log T$, is related to $\sigma$ by $\Delta \log T=1.67 \sigma$ The values of $\sigma$ and $\Delta \log T$ that we used are listed in Table 2.

The second form we consider is that of a step function:

$$
\varphi(\log \mathrm{T})= \begin{cases}A_{0} & \log T_{0}-\frac{1}{2} \Delta \leq \log T \leq \log T_{0}+\frac{1}{2} \Delta \\ 0 & \text { otherwise }\end{cases}
$$

Again, we adjust $A_{0}$ to maintain a constant integrated EM of $10^{27} \mathrm{~cm}^{-3}$. The range of $\Delta$ values we used is listed in Table 2 , while we choose $\log T_{0}=6.0$. These two distributions are chosen to investigate the case of a single, multithermal structure along the line of sight whose plasma is confined in a limited temperature range around a central value.

Equation 7 gives an approximate upper limit to the error $\Delta F$ expected for the observation of an isothermal plasma. For multithermal plasmas, we estimate $\Delta F$ using Monte Carlo simulations. We start with the $E M_{i}$ that would be obtained from 13 spectral lines with no errors, and then we perform a large number of simulated observations using $\triangle E M_{i} / E M_{i}$ errors selected randomly from a Gaussian distribution. The distribution has a standard deviation of either $10 \%$ or $40 \%$, corresponding to two different estimates of the measurement uncertainties. The spectral lines are assumed to have independent errors. For each simulation trial, we determine $F_{\text {min }}$ from the $F(T)$ curves. We do this for 10,000 trials and take the root mean square (RMS) deviation from the true value of $F_{\min }$ (obtained with no errors) as an estimate of the uncertainty $\Delta F$.

By repeating this procedure for many different assumed values of the plasma thermal width, we generate a curve of thermal width versus $F_{\min }$ with associated error bars $\Delta F$. An example is shown in Figure 2 for a Gaussian shaped thermal distribution. Black and gray curves represent $\triangle E M$ errors of $10 \%$ and $40 \%$, respectively. In each case the solid curve is the mean value of $F_{\text {min }}$ obtained from the Monte Carlo simulations, and the dashed curves indicate the $\pm \Delta F$ uncertainty. Note that the two solid curves are not the same and do not pass through the origin, as one might expect if they were the true values of $F_{\text {min }}$. They are not the true values, but rather the mean values. Differences arise because of the nonsymmetric shape of the $F_{\min }$ distributions, especially for plasmas that are close to isothermal. 
Plots like Figure 2 are very useful useful when we deal with plasmas that are either isothermal or are characterized by a narrow thermal distribution. In this case, functional forms like Gaussian distributions can be reasonable approximations to the real plasma thermal distribution, so that a comparison between the Monte Carlo simulations and the observed $F_{\min }$ value is a fast and effective way of evaluating the plasma distribution and its degree of multithermality. We simply examine a vertical line positioned at the appropriate $F_{\min }$ in Figure 2. Where it crosses the solid curve is the most likely value of the thermal width. The crossing points with the dashed curves indicate the range of acceptable thermal widths that are consistent with the observations given the assumed uncertainties. In many cases isothermal conditions will be allowed (when $F_{\min }$ is small and/or $\Delta F$ is large). Then, the crossing with the the upper dashed curve indicates the temperature resolution of the isothermal determination.

\section{Results}

\subsection{Gaussian distribution with variable width}

The Gaussian DEM widths listed in Table 2 allow us to simulate an increasingly multithermal distribution, as can be seen from the results displayed in Figure 3. The left column in Figure 3 displays the DEM curves used for each simulated spectrum; the $E M(T)$ curves are shown as a function of temperature in the right column. Figure 3 shows that, even with perfect data, analysis made "by eye" can lead to the conclusion that a plasma is isothermal even for thermal widths up to $\Delta \log T \approx 0.07$. The situation is of course more ambiguous in the presences of measurement errors. This demonstrates why a quantitative error analysis of the type we advocate here is so important.

Figure 2 was obtained with the same DEM curves of Figure 3. The assumed 10\% uncertainty in $\triangle E M$ (black curves) is very optimistic, while the assumed $40 \%$ uncertainty (red curves) is rather conservative. With the larger uncertainty, we find that the thermal width $\Delta \log T$ is uncertain by approximately 0.2 , depending on the value of $F_{\min }$. An isothermal interpretation is valid up to $F_{\min }=0.45$.

\subsection{Step distribution}

Figure 4 shows the application of the EM technique to the step-function DEM case. It shows that a "by eye" estimate indicates that the plasma might be isothermal up to an actual thermal width $\Delta=0.15$. This value can be interpreted as the smallest temperature width for 
which "by eye" estimates are able to distinguish an isothermal from a multithermal plasma when the DEM distribution is a step-function. Below that limit, the DEM of a multithermal plasma looks consistent with isothermal.

Figure 5 shows the corresponding thermal width versus $F_{\min }$ plot, again calculated with $10 \%$ and $40 \%$ uncertainties. The resulting uncertainties in the thermal width are somewhat larger than in the Gaussian case. An isothermal interpretation is once again valid up to about $F_{\min }=0.5$ for $40 \%$ uncertainties; however, a thermal width $\Delta=0.33$ is equally valid at this value. The ambiguity decreases for smaller uncertainties as well as for smaller $F_{\min }$.

\subsection{Importance of the number of lines}

The ability of the EM diagnostic technique at determining the thermal structure of a plasma is crucially determined by the amount and nature of the lines that are used. First, lines from the same ion have almost the same dependence on the electron temperature, so the information they convey is essentially the same. Thus, multiple lines from the same ion in the dataset help understanding whether any of them has any problem, but do not provide any substantial change to characteristics of the plasma thermal distribution determined using a single line from that ion.

Second, the difference of the temperature of formation of lines emitted by different ions is also of great importance. In fact, lines from ions that exist in the same temperature interval again provide somewhat similar information, and their combined use provides little benefit to the accuracy of the final results. On the contrary, lines from ions formed at very different temperature ranges provide the most benefit for the results.

To give an idea of the effect of lines from different ions, we have applied the EM diagnostic technique to subsets of the lines listed in Table 1 , and compared the resulting $\Delta \log T$ versus $F_{\text {min }}$ curves for Gaussian thermal distributions. Figure 6 shows the results for: case 1, which includes all 13 original lines (same as Fig. 2); case 2, where we omit lines formed at the lowest and highest temperatures; and case 3 , where we omit lines formed near $\log T=6.0$ in the middle of the temperature range.

Omitting lines formed in the middle of the range has only minimal impact on the diagnostics. However, omitting lines at the ends of the range and thereby limiting the temperature coverage has a very large impact. $\Delta F$ uncertainties are similar in the three cases, but because the curve for case 2 is much steeper, the uncertainty in thermal width at a given $F_{\min }$ is much larger. 


\subsection{Implementation}

It is very important to note that the relationship between the thermal width of the DEM distribution and $F_{m i n}$, as well as the calculation of the $F(T)$ curve and $\Delta F$ themselves, depend on the lines that are being used. Different sets of lines will provide different $F(T)$ curves, $F_{\min }$ and $\Delta F$ values. The present work does not set a definitive criterion to be blindly applied to any dataset, but rather it defines a method. $F(T), F_{\min }$ and $\Delta F$ need to be calculated for each data set of spectral lines for which the criteria we defined in this work will be applied. The recommended way to carry out an EM analysis is therefore to follow five steps:

1. Calculate an approximate upper limit of $\Delta F$ for an isothermal plasma using Equation 7 taking into account all the sources of uncertainty;

2. Calculate the $E M(T)$ curves for each line, and plot them versus temperature to visually check if a crossing point can be found;

3. Use the $E M(T)$ curves to calculate $F(T)$ and $F_{\text {min }}$, and compare $F_{\min }$ with $\Delta F$ to determine whether the plasma might be isothermal $\left(F_{\min } \leq \Delta F\right)$ or not;

4. Perform Monte Carlo simulations to obtain expected values of $F_{\min }$ using DEM distributions with a variety of input thermal widths, and generate a curve of thermal width verus $F_{\text {min }}$, including the error band as described in Section 2.3 (a copy of our IDL code written for this purpose is available upon request);

5. Compare the measured $F_{\min }$ with the curve and error band to determine the most likely thermal width of the observed distribution and the range of possible thermal widths that are allowed by the uncertainties.

Following these steps, the degree of multithermality of a plasma can be determined taking into account the uncertainties in the observations and in the atomic data, and the possibility of the plasma being isothermal can be checked unambiguously.

\section{Discussion and Conclusions}

In the present work we have investigated the potential of the EM diagnostic technique in order to provide a quantitative method to assess its ability to 1) indicate whether a plasma is isothermal or not, and 2) determine the range of widths of the plasma thermal distributions 
compatible with the observations. We have developed a diagnostic technique that allows us to measure the width of a multithermal distribution from a set of observed line intensities. We have tested it on simulated spectra, where all the parameters of the calculation were under control. However, the application of this technique to real solar spectra might face three problems: 1) availability of spectral lines; 2) uncertainties in measured intensities $I$ and contribution functions $G(T)$, and 3) element abundances.

Perhaps the worst problem is the availability of lines emitted by many different ions formed at widely different temperature ranges. We demonstrated that the diagnostic potential of this technique decreases substantially when the range of temperatures sampled by the available lines is narrow. For this reason, we 1) strongly recommend that the EM diagnostic technique be applied to datasets with a large number of lines from many ions that sample a wide temperature range, and 2) caution against results obtained by applying the EM technique to datasets consisting of the intensities of only few lines, or of narrow-band filters.

The other main source of uncertainty in the EM analysis is given by element abundances. In fact, in order to calculate the $\operatorname{EM}(T)$ and $F(T)$ curves it is necessary to adopt a set of element abundances which might not necessarily be the same as those in the emitting plasma. In this case, the crossing point will be less defined or even absent even if the plasma is strictly isothermal. This uncertainty can be avoided either by using lines emitted by ions of the same element, or by a class of elements whose relative abundances are known with high degree of accuracy and that do not change in different plasmas, such as elements with First Ionization Potential (FIP) smaller than $10 \mathrm{eV}$ (low-FIP elements), or larger than $10 \mathrm{eV}$ (high-FIP elements), in the solar corona.

We plan in a future paper to apply the present method to diagnostic results reported in the literature, in order to test the robustness of the claims of isothermality made in several works.

The work of E. Landi is supported by several NASA grants. The work of J.A. Klimchuk was supported by the NASA Living With a Star Program and by ONR. The idea for this study was born at a meeting of the International Space Science Institute (ISSI) working group on The Role of Spectroscopic and Imaging Data in Understanding Coronal Heating led by Dr. Susanna Parenti. 


\section{REFERENCES}

Brooks, D.H., Warren, H.p. Williams, D.R., Watanabe, T. 2009, ApJ, 705, 1522

Cargill, P.J., Klimchuk, J.A. 2004, ApJ, 605, 911

Dere, K.P., Landi, E., Mason, H.E., Monsignori Fossi, B.C., \& Young, P.R. 1997, A\&AS, 125,149

Dere, K.P., Landi, E., Young, P.R., Del Zanna,G., Landini, M., \& Mason, H.E. 2009, A\&A, 498,915

Feldman, U., \& Laming, J.M 2000, Phys. Scripta, 61, 222

Feldman, U., Mandelbaum, P., Seely, J.L., Doschek, G.A., \& Gursky H. 1992, ApJSS, 81, 387

Feldman, U., Doschek, G.A., Schühle, U., \& Wilhelm, K. 1999, ApJ, 518, 500

Judge, P.G. 2010, ApJ, 708, 1238

Klimchuk, J.A., Patsourakos, S., \& Cargill, P.J. 2008, ApJ, 682, 1351

Landi, E. \& Feldman, U. 2008, ApJ, 672, 674

Landi, E., Feldman, U., \& Dere, K.P. 2002, ApJS, 139, 281

Landi, E., Feldman, U., \& Doschek, G.A. 2006, ApJ, 643, 1258

Mazzotta, P., Mazzitelli, G., Colafrancesco, S., \& Vittorio, N. 1998, A\&AS, 133, 403

Patsourakos, S., \& Klimchuk, J.A. 2007, ApJ, 667, 591

Patsourakos, S., \& Klimchuk, J.A. 2009, ApJ, 696, 760

Phillips, K.J.H., Feldman, U., \& Landi, E. 2008, Ultraviolet and X-ray Spectroscopy of the Solar Atmosphere, Cambridge Astrophysics Series 44, Cambridge University Press, Cambridge, UK

Pottasch, S.R. 1963, ApJ, 137, 945

Warren, H.P. 1999, Solar Physics, 190, 363

Warren, H.P., Brooks, D.H. 2009, ApJ, 700, 762 


\begin{tabular}{lrc} 
Ion & Wvl. $(\AA)$ & $\log T_{\max }$ \\
\hline O v & 629.732 & 5.37 \\
Ne vI & 558.685 & 5.61 \\
Ne VII & 561.728 & 5.71 \\
Mg VII & 367.659 & 5.80 \\
& 367.672 & \\
Mg VIII & 315.016 & 5.90 \\
MgIX & 368.070 & 5.98 \\
MgX & 624.943 & 6.05 \\
SiXII & 520.666 & 6.28 \\
FeX & 345.735 & 5.98 \\
FeXI & 352.662 & 6.06 \\
FeXII & 364.467 & 6.14 \\
FeXIII & 320.809 & 6.20 \\
FeXIV & 334.180 & 6.27 \\
& & \\
\hline
\end{tabular}

Table 1: Lines considered in the present work, together with wavelength and temperature of maximum ion fractional abundance $\log T_{\max }$ (from Mazzotta et al. 1998).

\begin{tabular}{rrr|rc} 
& \multicolumn{3}{c}{ Gaussian } & \multicolumn{2}{c}{ Step function } \\
& \multicolumn{1}{c}{$\sigma$} & FWHM $(\Delta \log T)$ & $\Delta$ \\
\hline 1 & 0.032 & 0.053 & 1 & 0.20 \\
2 & 0.055 & 0.091 & 2 & 0.40 \\
3 & 0.077 & 0.13 & 3 & 0.60 \\
4 & 0.1 & 0.17 & 4 & 0.70 \\
5 & 0.17 & 0.29 & 5 & 0.80 \\
6 & 0.24 & 0.41 & 6 & 0.90 \\
7 & 0.32 & 0.53 & & \\
8 & 0.55 & 0.90 & & \\
& & & & \\
\hline
\end{tabular}

Table 2: Parameters for the DEM distributions. Left: widths $\sigma$ of the single Gaussian distribution, and corresponding half maximum widths $\Delta \log T$. Right: Width $\Delta$ of the step-function DEM. 


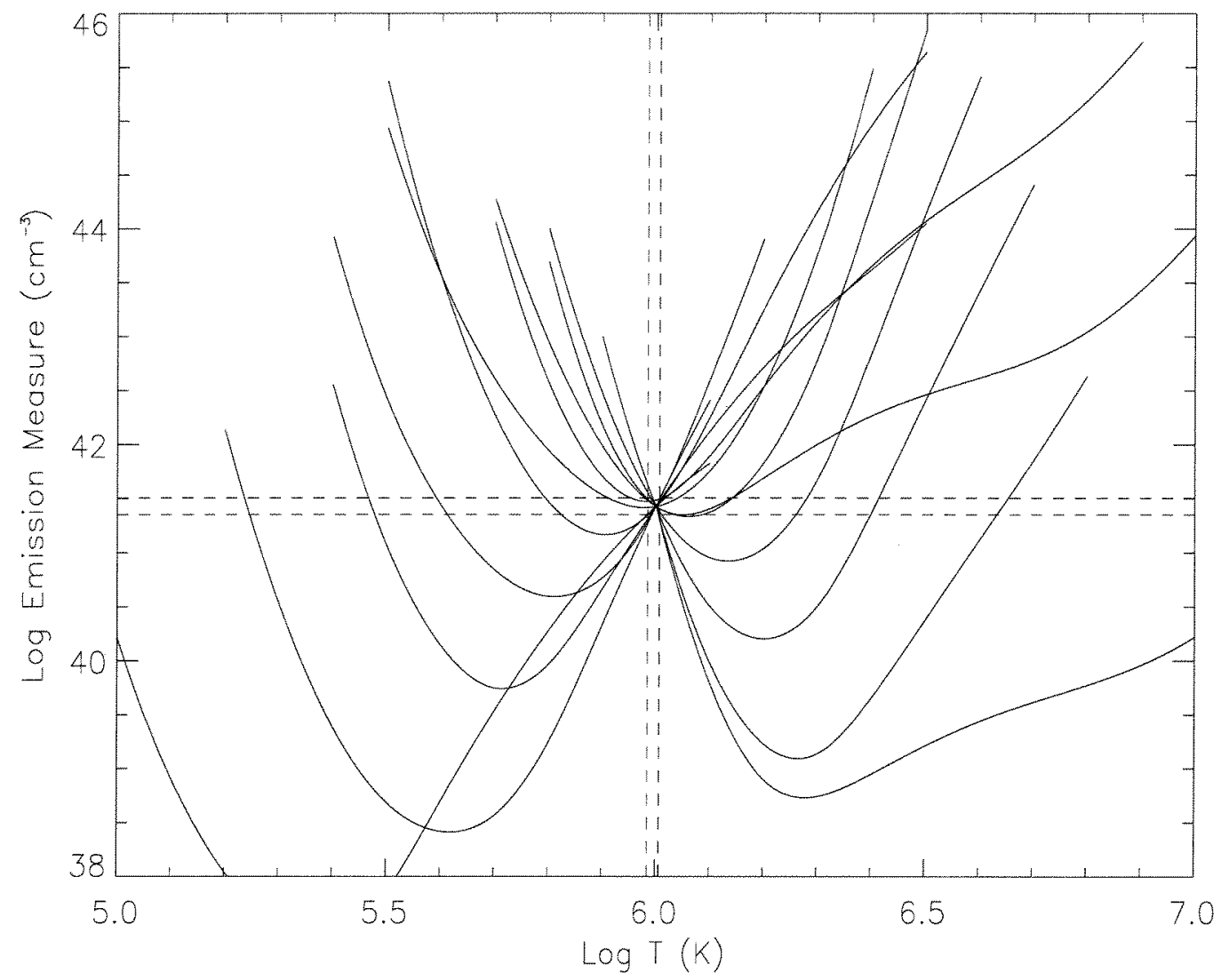

Fig. 1.- Example of the EM diagnostic technique applied to line intensities calculated assuming an isothermal spectrum (see text for details). The dashed lines indicate the range of $T$ and EM values where all the $E M(T)$ curves cross. 


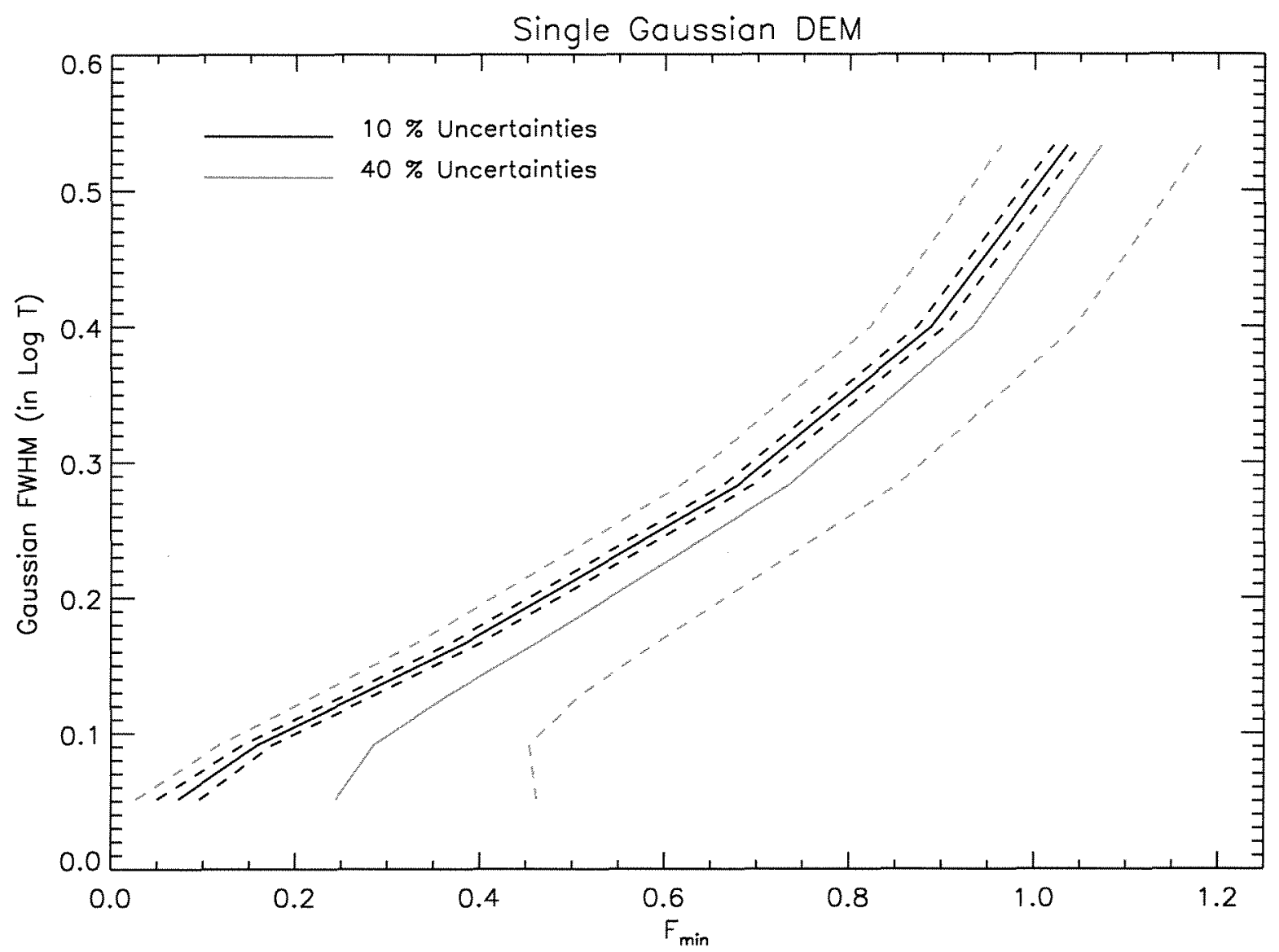

Fig. 2. - Thermal width $\Delta \log T$ versus $F_{m i n}$ for a Gaussian DEM thermal distribution. 

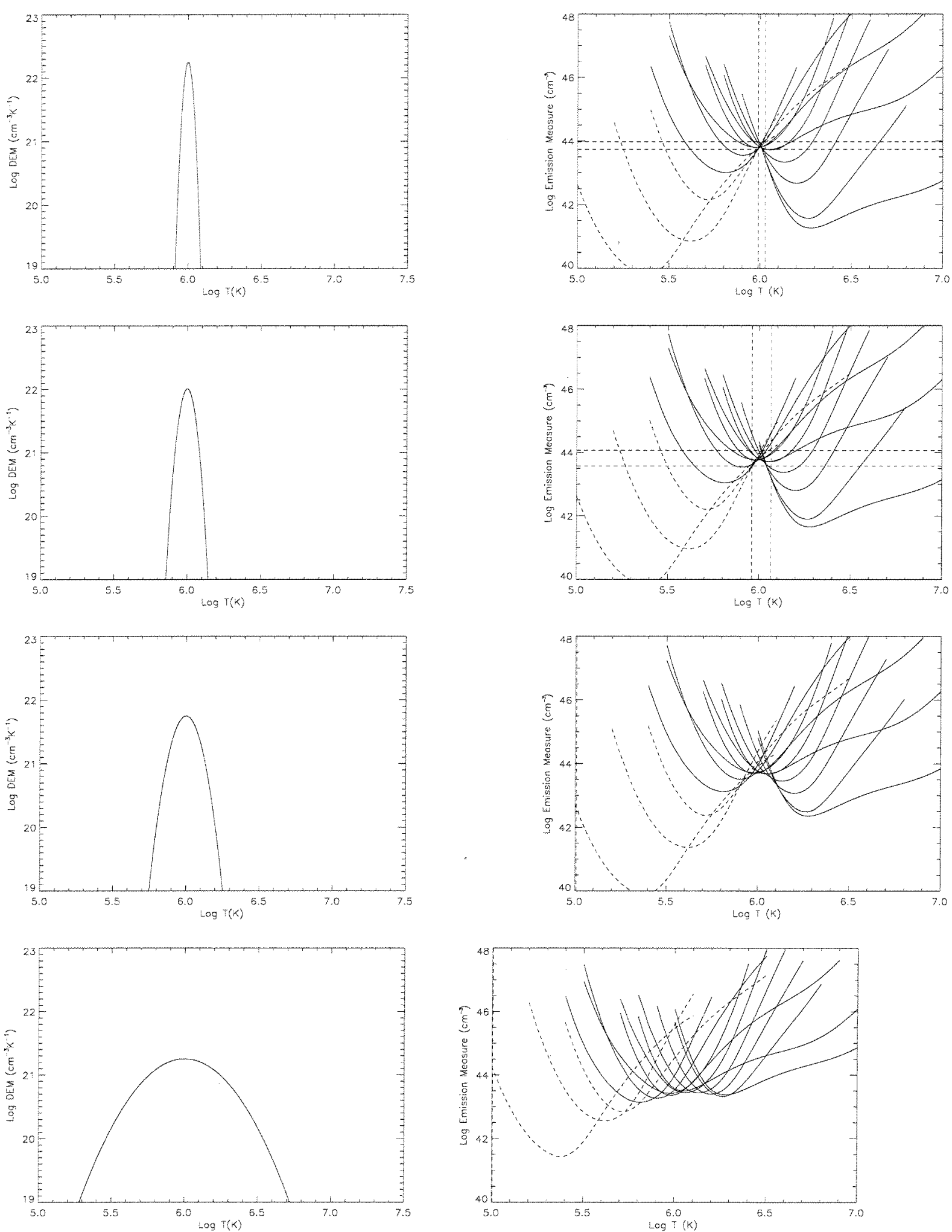

Fig. 3.- Left column: DEM curves used to calculate line intensities. Right column: $E M(T)$ curves obtained from the line intensities calculated using the DEM curves on the left. Values of $\sigma$ are (from top to bottom): $0.001,0.002,0.01$, and 0.1 , corresponding to $\Delta \log T=0.05,0.07,0.17$ and 0.53 . 

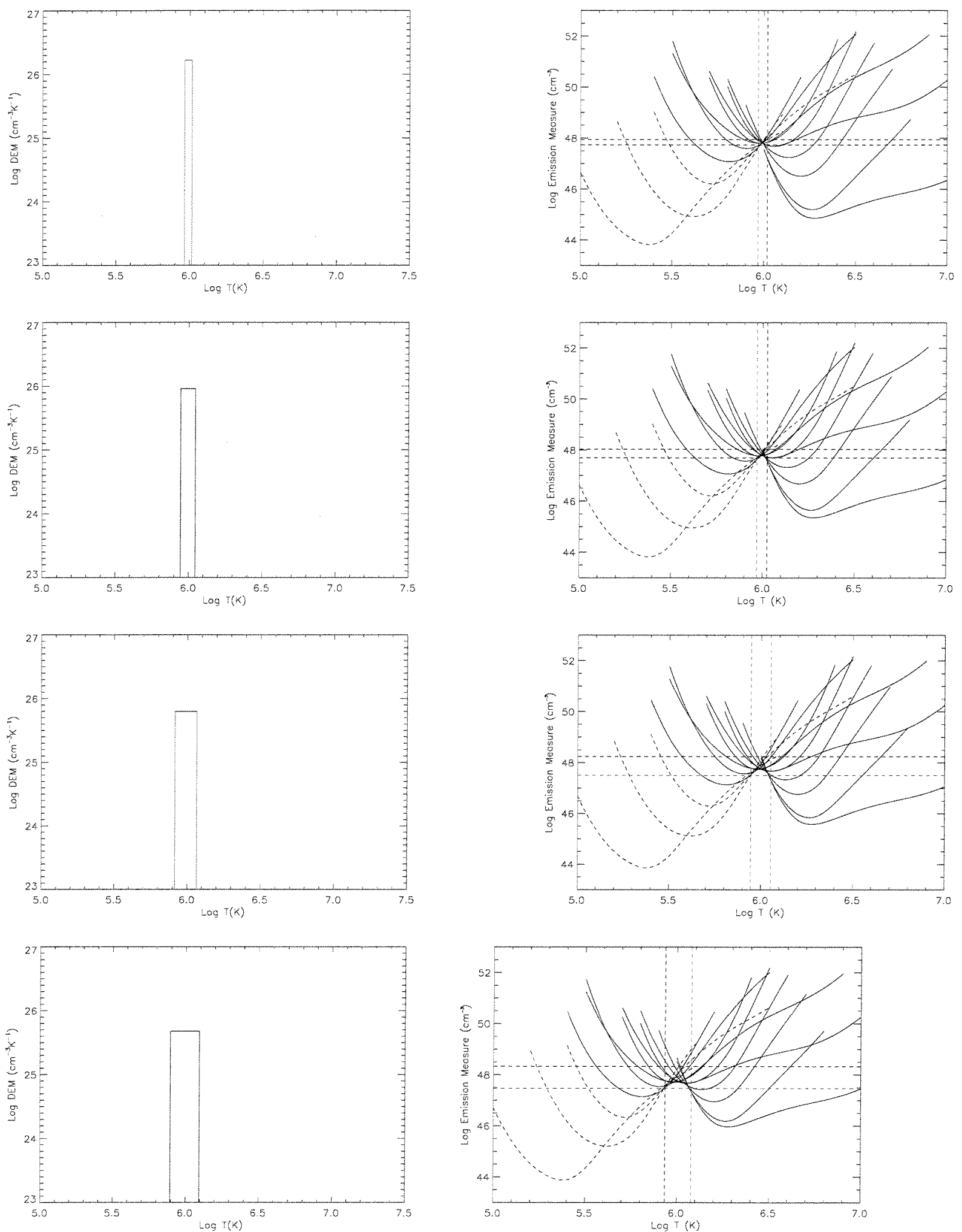

Fig. 4.- Left column: DEM curves used to calculate line intensities. Right column: $E M(T)$ curves obtained from the line intensities calculated using the DEM curves on the left. The $\Delta$ widths of the distributions are $0.05,0.1,0.15$, and 0.2 . 


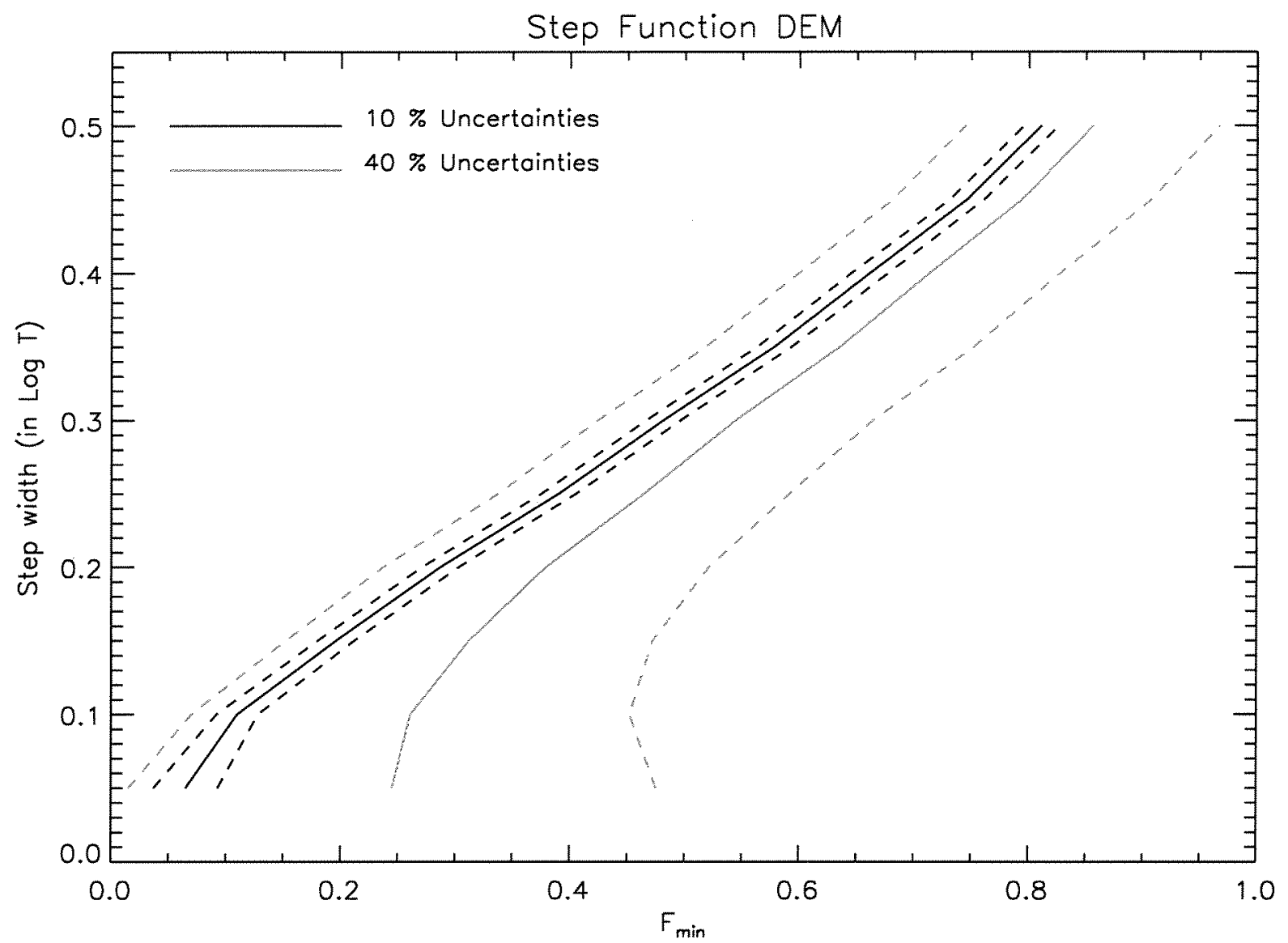

Fig. 5.- Thermal width $\Delta \log T$ versus $F_{\min }$ for a step function DEM thermal distribution. 


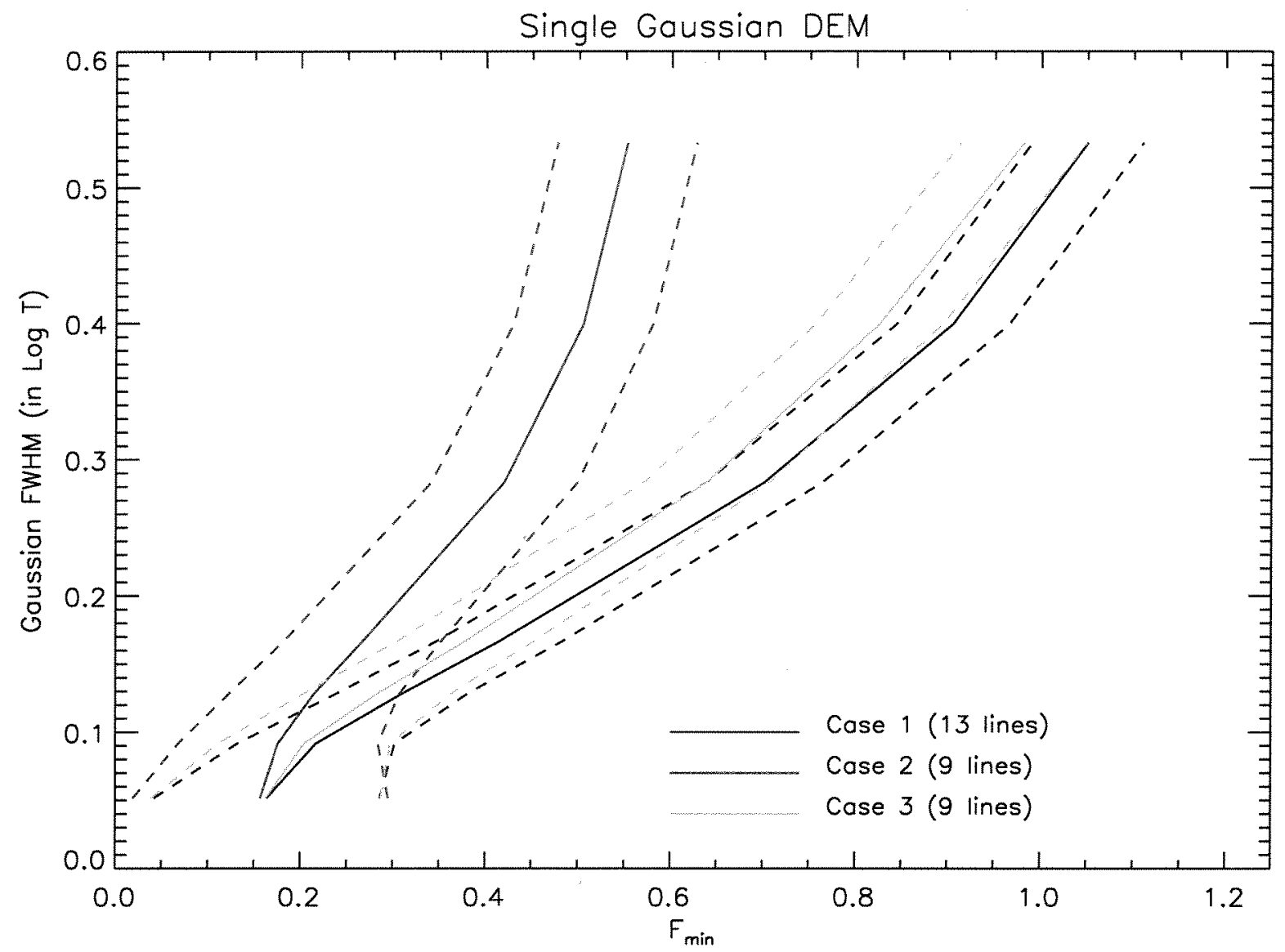

Fig. 6. - Thermal width $\Delta \log T$ versus $F_{\min }$ for a Gaussian DEM thermal distributions. Case 1: all 13 lines in Table 1. Case 2: 9 lines (Ov, Nevi, SixII, FexIV are omitted). Case 3: 9 lines (Mg IX,X, Fe X,XI are omitted). 